\title{
Highest Weight Representations of Quantum Current Algebras
}

\author{
Sergio Albeverio* and Shao-Ming Fei ${ }^{\star}$ \\ Institute of Mathematics, Ruhr-University Bochum, D-44780 Bochum, Germany \\ *SFB 237 (Essen-Bochum-Düsseldorf); BiBoS (Bielefeld-Bochum); \\ CERFIM Locarno (Switzerland)
}

\begin{abstract}
We study the highest weight and continuous tensor product representations of q-deformed Lie algebras through the mappings of a manifold into a locally compact group. As an example the highest weight representation of the q-deformed algebra $s l_{q}(2, \mathbb{C})$ is calculated in detail.
\end{abstract}

\footnotetext{
${ }^{1}$ Alexander von Humboldt-Stiftung fellow.

On leave from Institute of Physics, Chinese Academy of Sciences, Beijing
} 


\section{Introduction}

The structure and the representation theory of infinite-dimensional Lie algebras play important roles in mathematics and physics, especially in quantum field theory, and have been investigated for several decades by mathematicians and theoretical physicists. For instance, by generalizing Cartan's classification of simple Lie algebras, the Kac-Moody algebras can be built (by generators and relations) from a Cartan matrix, and possess a very rich representation theory. Among them is the class of affine Lie algebras, which can be realized as (untwisted or twisted) loop algebras, i.e., mappings from the circle $S^{1}$ into semisimple Lie algebras. For affine Lie algebras a large class of unitary highest weight representations can be classified, see e.g. [1].

Representations of q-deformed affine Lie algebras, so called quantum affine Lie algebras, have also been studied recently [2-4]. However the current algebra (also called gauge algebra) of the mappings from a manifold $M$, with $\operatorname{dim}(M)>1$, into Lie algebras are no longer Kac-Moody algebras (they do not possess a Cartan matrix). The motivation of this paper is to discuss the representation theory of such kind of q-deformed current algebras ${ }^{2}$.

Let $g$ be a Lie algebra of a locally compact group $G$. The current algebra with respect to $g$ is an algebra of mappings $g^{X}=\operatorname{Map}(X, g)$ from some manifold $X$ into $g$. The representation of this current algebra can be obtained from the representation of the related current group which are defined to be group of mappings $G^{X}=\operatorname{Map}(X, G)$ with its group structure provided by the pointwise multiplication law. For the representation of the current group, the continuous unitary representations in Hilbert space $\pi: G^{X} \rightarrow u(H)$ are of significance, where $u(H)$ denotes the unitary operators on a real Hilbert space $H$. Such representations provide a natural and nontrivial non-commutative generalization of the theory of distributions on $X \| \mathbb{1}$. Moreover, they correspond to the representations of the current algebra by self-adjoint operators, via the exponential mapping. That is, the representations of current algebras can be obtained as the infinitesimal representations of current groups.

One approach to obtain continuous unitary representations of current groups is to look

\footnotetext{
${ }^{2}$ we call them quantum current algebras, although the q-deformation and physical quantization are different in principle [5] [6]
} 
at the continuous tensor products of representations of $G$. Another approach is given by the highest weight representation theory [1]. For the group $G=S U(n, 1)$, and hence for the algebra $g=s u(n, 1)$, the connection between these two kinds of representations has been made by Torrésani []].

A difficulty concerning the extension of these concepts and relations to the case of q-deformed Lie algebras and groups lies in that the representations of the q-deformed Lie algebra can not be obtained as infinitesimal representations of quantum groups, in fact, the exponential mappings of the q-deformed Lie algebra do not give rise, in general, to representations of quantum groups. It is however interesting to ask the question whether the quantum current algebras have also continuous tensor product representations. In this letter we investigate the construction of continuous tensor product representations and show that in a particular case the connection can be made between continuous tensor product representations and highest weight representations of quantum current algebras.

\section{The continuous tensor product representation of q-deformed current algebras}

Let $A$ be a Lie algebra and $A_{q}$ the q-deformed Lie algebra of $A$ in the sense of [2] [8]. We denote by $B$ the Borel subalgebra of $A_{q}$ with an involutive antilinear antiautomorphism $\omega$ such that $B+\omega \cdot B=A$.

[Definition 1]. Let $\Lambda$ be a complex character of $B$, and $\pi: A_{q} \rightarrow \operatorname{End}(V)$ be a representation of $A_{q}$ such that there exists a vector $\nu_{\Lambda}$ in $V$ satisfying

$$
\pi(b) . \nu_{\Lambda}=\Lambda(b) \nu_{\Lambda}, \quad \pi(\omega \cdot b) \cdot \nu_{\Lambda}=V, \quad \forall b \in B
$$

Then $\pi$ is called a highest weight representation of $A_{q}$ with highest weight $\Lambda$.

[Definition 2]. Let $X$ be an m-dimensional manifold. The current algebra of $A$ is defined to be the algebra of continuous mappings

$$
A^{X}=\operatorname{Map}(X, A)
$$

from $X$ to $A$.

We define in $A_{q}$ a topology as in [2] [3]. 
[Definition 3]. Let $X$ be an m-dimensional manifold. The quantum current algebra of $A_{q}$ is defined to be the quantum algebra of continuous mappings

$$
A_{q}^{X}=\operatorname{Map}\left(X, A_{q}\right)
$$

from $X$ to $A_{q}$.

Let $X$ be a topological space and let $\mu$ be a finite positive measure. We first investigate the continuous tensor product of quantum current algebras and connect it to the highest weight representations. Set

$$
G_{t}=\left\{e^{t a} \mid a \in A_{q}\right\}, \quad t \in \mathbb{R}
$$

where $e^{t a}$ is defined by $e^{t a}=\sum_{n=0}^{\infty} \frac{t^{n}}{n !} a^{n}$. We call $G_{t}$ the $t$-exponential elements obtained from $A_{q}$ and the fixed parameter $t \in \mathbb{R}$.

Set

$$
G_{t}^{X}=\left\{e^{t \sigma} \mid \sigma \in A_{q}^{X}\right\}, \quad t \in \mathbb{R}
$$

where $e^{t \sigma}$ is defined pointwise by $e^{t \sigma}(x)=e^{t \sigma(x)}, \forall x \in X$. We call $G_{t}^{X}$ the t-exponential currents obtained from the quantum current algebra of $A_{q}$. We give $G_{t}$ the topology induced by the one defined in $A_{q}$.

Let $\left\{O_{x}\right\}_{x \in X}$ be an operator representation of $G_{t}$ acting on some Hilbert space $K_{X}$,

$$
O_{x}: G_{t} \rightarrow u\left(K_{X}\right)
$$

with $u\left(K_{X}\right)$ the unitary operators in $K_{X}$. Let $\left\{b_{x}\right\}_{x \in X}$ be an associated continuous $\mu$ measurable field of one-cocycles,

$$
b_{x}: G_{t} \rightarrow K_{X}
$$

satisfying

$$
b_{x}\left(g \cdot g^{\prime}\right)=b_{x}(g)+O_{x}(g) b_{x}\left(g^{\prime}\right), \quad g, g^{\prime} \in G_{t}
$$

such that for all $x$ in $X, b_{x}\left(G_{t}\right)$ is total in $K_{X}$. For an example of such a cocycle we refer to section 3. For all $f$ in $G_{t}^{X}$, we set

$$
\begin{gathered}
\tilde{O}(f)=\int_{X}^{\oplus} O_{x}[f(x)] d \mu(x) \\
\tilde{b}(f)=\int_{X}^{\oplus} b_{x}[f(x)] d \mu(x)
\end{gathered}
$$




$$
\tilde{K}=\int_{X}^{\oplus} K_{X}(x) d \mu(x)
$$

with $K_{X}(x)$ the $x$-copy of $K_{X}$. $\tilde{O}$ is a linear representation of $G_{t}$. The integrals have to be understood as direct integrals, see e.g. [9].

Let $\tilde{H}$ be the Fock space over $\tilde{K}$.

$$
\tilde{H}=\operatorname{Exp}[\tilde{K}] .
$$

By construction the coherent states

$$
\operatorname{Exp}[\tilde{k}]=\sum_{n=0}^{\infty} \frac{\tilde{k}^{\otimes n}}{n !}, \quad \tilde{k} \in \tilde{K}
$$

are total in $\operatorname{Exp}[\tilde{K}]$, see e.g. [9]. The representation $\tilde{u}=\operatorname{Exp}[\tilde{O}]$ is then defined by the action on $\operatorname{Exp}[\tilde{K}]$,

$$
\tilde{u}(f) \cdot \operatorname{Exp}[h]=e^{-\|\tilde{b}(f)\|^{2} / 2-<\tilde{O}(f) \cdot h, \tilde{b}(f)>} \operatorname{Exp}[\tilde{O}(f) \cdot h+\tilde{b}(f)],
$$

where $h \in \tilde{K} .<,>$ denotes the scalar product in $\tilde{K}$ inherited from the one defined in the Hilbert space $K_{x}, x \in X$, i.e.

$$
<\tilde{k}, \tilde{k}^{\prime}>=\int_{X}^{\oplus}<k_{x}, k_{x}^{\prime}>d \mu(x), \quad \forall \tilde{k}, \tilde{k^{\prime}} \in \tilde{K} .
$$

For $\sigma \in A_{q}^{x}$, we define a representation $\pi$ of $\sigma$ in $\operatorname{Exp}[\tilde{K}]$ by

$$
\pi(\sigma)=\left\{\frac{d}{d t} \tilde{u}\left(f_{t}\right)\right\}_{t=0},
$$

where $f_{t}=e^{t \sigma} \in G_{t}^{X}$. Note that the derivative exists since $\tilde{u}$ is an algebraic representation and $t \rightarrow f_{t}$ is also smooth by construction.

[Proposition 1]. $\pi$ acts as follows on the coherent vectors:

$$
\begin{aligned}
\pi(\sigma) \cdot \operatorname{Exp}[h]= & \left(-i \tilde{\phi}(\sigma)-<h, \tilde{\nu}_{\sigma}>\right) \operatorname{Exp}[h] \\
& +\sum_{n=0}^{\infty} \frac{n+1}{\sqrt{(n+1) !}}\left[\tilde{\theta}(\sigma) \cdot h+\tilde{\nu}_{\sigma}\right] \otimes h^{\otimes n}, \quad \forall h \in \tilde{K},
\end{aligned}
$$

where

$$
\begin{aligned}
\tilde{\theta}(\sigma) & =\left\{\frac{d}{d t} \tilde{O}\left(f_{t}\right)\right\}_{t=0} & \in \operatorname{End}(\tilde{K}) \\
\tilde{\nu}_{\sigma} & =\left\{\frac{d}{d t} \tilde{b}\left(f_{t}\right)\right\}_{t=0} & \in \tilde{K} \\
\tilde{\phi}(\sigma) & =\left\{\frac{d}{d t} \tilde{\psi}\left(f_{t}\right)\right\}_{t=0} & \in \mathbb{C}
\end{aligned}
$$


$\pi$ is a unitary representation of $G_{t}^{X}$ on the Fock space $\tilde{H}$. Moreover, if $\mu$ is non-atomic, $\pi$ is irreducible.

[Proof]. This is a direct consequence of definitions of (5) and (6) using methods in [7].

\section{The case of the q-deformed current algebra $s l_{q}(2, C)^{X}$}

Now we take for $A_{q}$ the quantum algebra $A_{q}=s l_{q}(2, \mathbb{C})$, as an example. Let $e_{0}, f_{0}$ and $h_{0}$ be the generators of the algebra $A=\operatorname{sl}(2, \mathbb{C})$, satisfying

$$
\left[h_{0}, e_{0}\right]=e_{0}, \quad\left[h_{0}, f_{0}\right]=-f_{0}, \quad\left[e_{0}, f_{0}\right]=2 h_{0}
$$

Let $e, f$ and $h$ be the corresponding elements of the quantum algebra $s l_{q}(2, \mathbb{C}$ ) (for the study of this algebra see, e.g. [2] 8]) satisfying the commutation relations

$$
[h, e]=e, \quad[h, f]=-f, \quad[e, f]=[2 h]_{q} .
$$

where $[x]_{q}=\frac{q^{x}-q^{-x}}{q-q^{-1}}$ and $q \in \mathbb{R} \backslash\{0\}$ is the deformation parameter.

The following mapping is a deformation map from $s l(2, \mathbb{C})$ to $s l_{q}(2, \mathbb{C})$.

$$
e=e_{0} \frac{[j-h]_{q}}{j-h}, \quad f=f_{0} \frac{[j+h]_{q}}{j+h}, \quad h=h_{0},
$$

where $j$ is the center of $\operatorname{sl}(2, \mathbb{C})$. It is straightforward to verify that $e, f, h$ satisfy the $s l_{q}(2, \mathbb{C})$ algebraic relations (12) in terms of relations (11) [6] [10].

Let $e_{0}(x), f_{0}(x), h_{0}(x)$ be the generators of the current algebra $s l(2, \mathbb{C})^{X}$ and $e(x)$, $f(x), h(x)$ the corresponding elements of the quantum current algebra $s l_{q}(2, \mathbb{C})^{X}$. Here and in the following $x$ runs over $X$.

[Proposition 2]. The deformation map from $\operatorname{sl}(2, \mathbb{C})^{X}$ to $s l_{q}(2, \mathbb{C})^{X}$ is given by

$$
e(x)=e_{0}(x) \frac{[j(x)-h(x)]_{q}}{j(x)-h(x)}, \quad f(x)=f_{0}(x) \frac{[j(x)+h(x)]_{q}}{j(x)+h(x)}, \quad h(x)=h_{0}(x), \quad \forall x \in X,
$$

where $j(x)$ is the center of $\operatorname{sl}(2, \mathbb{C})^{X}$.

[Proof]. The result is obvious from the map (13) and the fact that the current algebra is given the pointwise algebraic structure. Hence the deformation mapping $A$ to $A_{q}$ directly gives rise to the deformation mapping from $A^{X}$ to $A_{q}^{X}$. 
Therefore the map from a topological space $X$ to $A_{q}^{X}$ can be obtained from the maps $X$ to $A^{X}$ and $A^{X} \rightarrow A_{q}^{X}$.

Now let us take $X=D$ with $D$ the unit disk of the complex plane and consider the space $K=L_{h o l}^{2}(D, \mu), L_{h o l}^{2}$ being the space of square integrable holomorphic function on $D, \mu$ being the Riemannian volume $\mu(d x)=r d r d \theta, x=r e^{i \theta}, r \in \mathbb{R}_{+}, \theta \in[0,2 \pi]$. The generators of $s l(2, \mathbb{C})^{D}$ are

$$
e_{0}(\xi)=\left(\begin{array}{cc}
0 & \xi \\
0 & 0
\end{array}\right), \quad f_{0}(\xi)=\left(\begin{array}{cc}
0 & 0 \\
\xi & 0
\end{array}\right), \quad h_{0}(\xi)=\frac{1}{2}\left(\begin{array}{cc}
\xi & 0 \\
0 & -\xi
\end{array}\right), \quad \xi \in C_{0}^{\infty}(D)
$$

with the center $j \rightarrow j(\xi)=\left(\begin{array}{ll}\xi & 0 \\ 0 & \xi\end{array}\right)$, cfr. [0].

From the map (113) we have the elements of $s l_{q}(2, \mathbb{C})^{D}$,

$$
e(\xi)=\frac{\sinh \gamma \xi}{\sinh \gamma}\left(\begin{array}{ll}
0 & 1 \\
0 & 0
\end{array}\right), \quad f(\xi)=\frac{\sinh \gamma \xi}{\sinh \gamma}\left(\begin{array}{ll}
0 & 0 \\
1 & 0
\end{array}\right), \quad h(\xi)=\frac{1}{2}\left(\begin{array}{cc}
\xi & 0 \\
0 & -\xi
\end{array}\right),
$$

where $\gamma=\log q$ is the deformation parameter.

For $g=\left(\begin{array}{cc}a & b \\ c & d\end{array}\right) \in G_{t}, a, b, c, d \in C_{0}^{\infty}(D)$, the representation $O_{x}: G \rightarrow u(K), x \in D$, is defined by $O_{x}(g): f \in K \rightarrow O_{x}(g) . f$,

$$
\left[O_{x}(g) . f\right](z)=[-c z+a]^{-2} f\left(\frac{d z+b}{-c z+a}\right), \quad \forall z \in D .
$$

The $K$-valued one-cocycle is unique up to equivalence and is given by

$$
\left[b_{x}(g)\right](z)=\frac{c}{a-c z}, \quad \forall z \in D .
$$

For $f_{t}=e^{t \sigma}, \sigma=\left(\begin{array}{ll}\alpha & \beta \\ \gamma & \delta\end{array}\right) \in s l_{q}(2, \mathbb{C})^{D}$, we have that the quantity (9) is given by

$$
\begin{aligned}
\tilde{\nu}_{\sigma}(z) & =\left\{\frac{d}{d t} \tilde{b}\left(f_{t}\right)(z)\right\}_{t=0} \\
& =\left\{\frac{d}{d t} \int_{D}^{\oplus} b_{x}\left[f_{t}(x)\right](z) d \mu(x)\right\}_{t=0} \\
& =\int_{D}^{\oplus} \gamma(x) d \mu(x) \lambda_{0}(z), \quad z \in D
\end{aligned}
$$

with $\lambda_{0}$ the unit function on $D$ defined by $\lambda_{0}(z)=1$. Correspondingly $\tilde{\phi}$, occurring in (10), is here given by

$$
\tilde{\phi}(\sigma)=\left\{\frac{d}{d t} \tilde{\psi}\left(f_{t}\right)\right\}_{t=0}=\int_{D} \alpha(x) d \mu(x) .
$$


[Proposition 3]. Let $\pi$ defined by (7) as a representation of $s l_{q}(2, \mathbb{C})^{D}$ in $\operatorname{Exp}[\tilde{K}]$, $\tilde{K} \equiv \int_{D}^{\oplus} K_{D}(x) d \mu(x)$. Then $\pi$ is a highest weight representation of $s_{q}(2, \mathbb{C})^{D}$, and its highest weight is completely determined by the Riemannian volume $\mu$ on $D$.

[Proof]. We have only to check that $\pi$ admits a highest weight vector. Let $\Omega=\operatorname{Exp}[0]$, where 0 is the zero element in $\tilde{K}$. From (7), (16), (19) and (20) we have

$$
\begin{aligned}
\pi(e(\xi)) & =0 \\
\pi(f(\xi)) & =\int_{D}^{\oplus} \frac{\sinh \gamma \xi(x)}{\sinh \gamma} d \mu(x) \lambda_{0} \\
\pi(h(\xi)) & =\left[-i \int_{D} \frac{\sinh \gamma \xi(x)}{\sinh \gamma} d \mu(x)\right] . \Omega
\end{aligned}
$$

Therefore from the definition $1, \Omega$ is a highest weight vector for $\pi$. The corresponding highest weight of the quantum current algebra $s l_{q}(2, \mathbb{C})^{D}$ is completely determined by the $\mu$ measure on $D$.

We remark that when the deformation parameter $\gamma$ approaches zero, formulae (21) become the ones of $\operatorname{sl}(2, \mathbb{C})^{D}$ (in the sense that the formal replacement of $\gamma$ by zero in (21) yields the formulae in [7]). Hence our representation is a q-extension of the representation of $\operatorname{sl}(2, \mathbb{C})^{D}$ obtained in [7].

Summarizing, we have discussed the representations of quantum current algebras defined by mappings from manifolds into quantum algebras. This is a continuous tensor product representation. It is shown that for the quantum current algebra $\operatorname{sl}_{q}(2, \mathbb{C})^{D}$, the continuous tensor product representation is also a highest weight representation.

Remark: We have taken the Hilbert space to be real in this paper. Our construction can be extended to the case where the Hilbert space is complex, by changing the definition (6) by a factor corresponding to a nonvanishing 2-cocycle [1] [0].

ACKNOWLEDGEMENTS: We are very grateful to Professor Torrésani for very helpful discussions in connection with his work which has been the basis for our own work. We thank A.v. Humboldt foundation for the financial support given to the second named author.

\section{References}


[1] S. Albeverio, R. Høegh-Krohn, J. Marion, D. Testard and B. Torrésani, Noncommutative Distributions, Unitary Representation of Gauge Groups and Algebras, Monographs and Textbooks in Pure and Applied Mathematics 175, 1993.

[2] M. Jimbo, Lett. Math. Phys. 10(1985)63; 11(1986)247; Comm. Math. Phys. 102 (1986) 537.

[3] I.B. Frenkel, N.Yu. Reshetikhin, Commun. Math. Phys. 146(1992)1-60.

[4] G. Delius and Y.Z. Zhang, Finite Dimensional Representations of Quantum Affine Algebras, hep-th/9403162.

[5] M Flato and Z. Lu, Lett. Math. Phys. 21(1991)85;

M. Flato and D. Sternheimer, Lett. Math. Phys. 22(1991)155.

[6] S.M. Fei, J. Phys. A 24(1991)5195-5214.

S.M. Fei and H.Y. Guo, Commun. Theor. Phys. 20(1993)299-312.

[7] B. Torrésani, Unitary Highest Weight Representations of Gauge Groups, in S. Albeverio, J.E. Fenstad, H. Holden, T. Lindstr $\phi m$, Eds. Ideas and Methods in Mathematical Analysis, Stochastics, and Applications, Vol. I, pp.332-343, Cambridge University Press, 1992.

[8] V.G. Drinfeld, Sov. Math. Dokl. 32(1985)254; Proc. I.C.M. Berkeley (1986)798-820; L.D. Faddeev, Leningrad Math. J. Vol.1 (1990), No.1.

[9] A. Guichardet, Symmetric Hilbert space and related topics, LN Math 261, Springer, $\operatorname{Berlin}(1972)$.

[10] T.L. Curtright, G.I. Ghandour and C.K. Zachos, J. Math. Phys. 32(1991)3;

T.L. Curtright and C.K. Zachos, Phys. Lett. B 243(1990)237. 